\title{
Toxicidad de spinetoram y matrine sobre los estados de desarrollo de dos enemigos naturales: Chrysoperla externa y Ceraeochrysa cincta (Neuroptera: Chrysopidae)
}

Alegre, A., Joyo, G. E. y lannacone, J.

DOI: $10.31047 / 1668.298 x . v 38 . n 1.30498$

\section{RESUMEN}

El manejo integrado de plagas (MIP) requiere la compatibilidad del control químico y control biológico. El objetivo de esta investigación fue evaluar la toxicidad de los insecticidas spinetoram y matrine sobre los estados de desarrollo de dos enemigos naturales: Chrysoperla externa y Ceraeochrysa cincta. Se realizaron ensayos toxicológicos con spinetoram y matrine. Los productos fitosanitarios no afectaron la eclosión de los huevos y la emergencia de las pupas de ambas especies mediante ensayos de inmersión. Se obtuvieron altos porcentajes de mortalidad en adultos por contacto residual al usar spinetoram. Los adultos mostraron mayor susceptibilidad que el estado larval mediante ensayos de ingestión. Según la catalogación toxicológica para ensayos de laboratorio por contacto residual, la máxima concentración evaluada de spinetoram fue ligeramente nociva para larvas del primer y segundo estadio de C. externa, y ambas concentraciones fueron ligeramente nocivas para adultos de $C$. externa. Asimismo, ambas dosis fueron moderada y ligeramente nocivas en adultos de C. cincta. Finalmente, ambos productos fueron inofensivos en todos los estadios larvales de $C$. externa y $C$. cincta, mientras que el spinetoram fue ligeramente nocivo en adultos de ambas especies y solo la máxima concentración de matrine fue ligeramente nociva en adultos de $C$. cincta.

Palabras clave: controladores biológicos; crisopas; control químico; productos fitosanitarios; mortalidad.

Alegre, A., Joyo, G. E. and lannacone, J., 2021. Toxicity of spinetoram and matrine on the development stages of two natural enemies: Chrysoperla externa and Ceraeochrysa cincta (Neuroptera: Chrysopidae). Agriscientia 38: 39-50

\section{SUMMARY}

Integrated Pest Management (IPM) requires the compatibility of chemical control and biological control. The objective of this research was to evaluate the toxicity 
of spinetoram and matrine insecticides on the development stages of two natural enemies: Chrysoperla externa and Ceraeochrysa cincta. Toxicological tests were carried out with spinetoram and matrine. The phytosanitary products did not affect the hatching of the eggs and the emergence of the pupae of both species through immersion tests. High percentages of mortality were obtained in adults by residual contact when using the spinetoram. Adults showed greater susceptibility than the larval stage by ingestion tests. According to the toxicological cataloguing for laboratory test by residual contact, the highest concentration of spinetoram evaluated was slightly harmful for larvae of the first and second stage of $C$. externa and both concentrations were slightly harmful for $C$. externa adults. Likewise, both doses were moderately and slightly harmful in adults of $C$. cincta. Finally, both products were harmless in all larval stages of C. externa and $C$. cincta, while spinetoram was slightly harmful in adults of both species and only the highest concentration of matrine was slightly harmful in $C$. cincta adults.

Key Words: biological controllers; lacewings; chemical control; phytosanitary products; mortality.

Alegre, A. (ORCID: 0000-0002-3197-5674) y Joyo, G. E. (ORCID: 00000003-4785-5105): Universidad Nacional Agraria La Molina (UNALM), Facultad de Agronomía, Departamento Académico de Entomología. La Molina, Lima, Perú. lannacone, J. (ORCID: 0000-0003-36994732): Universidad Nacional Federico Villarreal (UNFV), Facultad de Ciencias Naturales y Matemática (FCCNM), Escuela Universitaria de Posgrado, Grupo de Investigación en Sostenibilidad Ambiental (GISA), Laboratorio de Ecología y Biodiversidad Animal (LEBA). El Agustino, Lima, Perú. Universidad Científica del Sur. Carrera de Ingeniería Ambiental, Laboratorio de Ingeniería Ambiental. Villa El Salvador, Lima, Perú. Correspondencia a: joseiannaconeoliver@gmail.com

\section{INTRODUCCIÓN}

Los productos fitosanitarios, como los insecticidas, herbicidas y acaricidas, representan herramientas de gran utilidad para el manejo de cultivos y desempeñan un rol importante en la producción agrícola mundial (Topping, Aldrich y Berny, 2020). Sin embargo, su uso excesivo en la agricultura puede provocar contaminación ambiental y afectar a los enemigos naturales, favoreciendo el resurgimiento de plagas, la aparición de plagas secundarias y el desarrollo de plagas resistentes (Baker, Green y Loker, 2020). Debido a esto, para mantener la sostenibilidad de la agricultura a mediano y largo plazo, el manejo integrado de plagas (MIP) representa una alternativa al control profiláctico de plagas, teniendo en cuenta el uso racional de productos fitosanitarios y la integración de diferentes estrategias de control
(Jindal, Dhaliwal y Koul, 2013), como por ejemplo, el uso de los insecticidas bioracionales o de riesgo reducido, los cuales son compuestos sintéticos o naturales que controlan eficientemente las plagas, sin producir un riesgo para los organismos no blanco (humanos, animales y enemigos naturales) y el ambiente (Topping et al., 2020).

El insecticida spinetoram es una mezcla de dos spinosinas semisintéticas (spinosina $J$ y spinosina L), las cuales son metabolitos secundarios de la bacteria Saccharopolyspora spinosa (Mertz \& Yao) (Dripps et al., 2011). El ingrediente activo afecta el receptor nicotínico de la acetilcolina y al receptor de GABA (Fu et al., 2018). Este insecticida se usa para el control de Aphis spiraecola Patch (Hemiptera: Aphididae) en el cultivo de granada; Thrips tabaci Lindeman (Thysanoptera: Thripidae) en espárrago; Panonychus citri McGregor (Prostigmata: Tetranychidae) en mandarina; Oligonychus 
punicae Hirst (Prostigmata: Tetranychidae) en palto; Bemisia tabaci (Gennadius) (Hemiptera: Aleyrodidae); Tetranychus urticae C. L. Koch (Prostigmata: Tetranychidae), Planococcus ficus Signoret (Hemiptera: Pseudococcidae) en vid; Polyphagotarsonemus latus (Banks) (Trombidiformes: Tarsonemidae) en pimiento; Chaetanaphothrips signipennis (Bagnall) (Thysanoptera: Thripidae) en plátano/banano y $B$. tabaci en arándano (Sistema Integrado de Gestión de Insumos Agropecuarios [SIGIA], 2009). Por otro lado, el insecticida matrine es un alcaloide quinolizidínico extraído de las raíces secas de Sophora flavescens Aiton, S. tonkinensis Gagnep. y S. alopecuroides L. (Huangy Xu, 2016). El ingrediente activo afecta al canal de sodio en el sistema nervioso del insecto (Du, Li, He y Liu, 2004). Este insecticida se usa para el control de T. tabaci en el cultivo de cebolla; Prodiplosis longifila Gagne (Diptera: Cecidomyiidae) en papa; Chloridea virescens (Fabricius) (Lepidoptera: Noctuidae) en alcachofa y algodón; Plutella xylostella L. (Lepidoptera: Plutellidae) en brócoli, Tallula atramentalis (Lederer) (Lepidoptera: Pyralidae) en granada; Spodoptera frugiperda (Smith) (Lepidoptera: Noctuidae) y P. longifila en espárrago; Phyllocnistis citrella Stainton (Lepidoptera: Gracillariidae) en mandarina; Oiketicus kirbyi Guilding (Lepidoptera: Psychidae) en palto; T. tabaci en vid, S. frugiperda en maíz y pimiento, $P$. longifila en pimiento y $C$. virescens en arándano (SIGIA, 2009).

Entre los organismos benéficos de importancia agrícola, los enemigos naturales de los artrópodos desempeñan una función importante en la regulación de densidades/poblaciones de insectos plagas (Bueno, Carvalho, Santos, Sosa-Gómez, y Silva, 2017). Las especies Chrysoperla externa (Hagen) y Ceraeochrysa cincta (Schneider) de la familia Chrysopidae (Neuroptera) son de amplia distribución en el Perú y se destacan por sus características depredadoras, presencia de adultos a través de todo el año, fácil crianza en condiciones de laboratorio, potencial de adaptación en diferentes agroecosistemas y su resistencia a numerosos productos fitosanitarios, motivo por el cual son considerados como enemigos naturales promisorios en el MIP en cultivos de maíz, papa, algodón, palma aceitera y frutales (cítricos, olivo y manzano) (Núñez, 1988a). C. externa es oófago y larvífago por naturaleza, predominando en cultivos de maíz, alimentándose de $S$. frugiperda, S. eridania (Lepidoptera: Noctuidae) y áfidos, mientras que $C$. cincta es depredador de ninfas de mosca blanca, arañuelas, cochinillas, predominantes en cultivos de cítricos, palto, olivo y algodón (Núñez, 1988b).

Los productos fitosanitarios más apropiados para un MIP son aquellos que combinan un control óptimo de las plagas objetivo con un mínimo impacto en los enemigos naturales (Baker et al., 2020); sin embargo, el impacto de muchos de estos productos desarrollados en la actualidad es aún poco conocido (Depalo, Lanzoni, Masetti, Pasqualini y Burgio, 2017). A la fecha, no se tiene información publicada sobre los efectos tóxicos del spinetoram y matrine sobre los crisópidos depredadores. Por tal motivo, la finalidad del presente trabajo fue evaluar la toxicidad o la selectividad de ambos productos fitosanitarios sobre los estados de desarrollo de dos enemigos naturales: $C$. externa y $C$. cincta, para su uso seguro en condiciones de campo en el marco de un manejo integrado de plagas (MIP).

\section{MATERIALES Y MÉTODOS}

Los ensayos de toxicidad se realizaron en el Laboratorio de Ecología y Biodiversidad Animal (LEBA) de la Facultad de Ciencias Naturales de la Universidad Nacional Federico Villareal (UNFV), Lima, Perú, mientras que la cría se realizó en el Laboratorio de Control Biológico de la Facultad de Agronomía de la Universidad Nacional Agraria La Molina (UNALM), Lima, Perú.

\section{Material biológico y cría}

Los huevos (1 millar) de $C$. externa y $C$. cincta dispuestos en tubos de cartulina y huevos congelados a granel $(10 \mathrm{~g})$ de Sitotroga cerealella Oliver (Lepidoptera: Noctuidae) se obtuvieron del Programa Nacional de Control Biológico del Servicio Nacional de Sanidad Agraria (SENASA). Se usaron recipientes plásticos $(36 \times 28 \times 18 \mathrm{~cm})$ para la cría de larvas, las cuales fueron alimentadas ad libitum con huevos de S. cerealella (Lepidoptera: Noctuidae). Adicionalmente, se colocaron en la base de los recipientes cinco tiras de cartón corrugado $(20 \times 3 \mathrm{~cm})$ para que las larvas pupen. Las pupas se colocaron en el interior de una jaula de dos mangas hasta la emergencia de los adultos (SENASA, 2015).

\section{Productos fitosanitarios}

Se emplearon un producto químico de uso agrícola (PQUA): spinetoram (Absolute ${ }^{\mathrm{TM}} 60 \mathrm{SC}$, $60 \mathrm{~g} / \mathrm{l}^{-1}$, Dow Agrosciences, Perú), y un producto biológico de uso agrícola (PBUA): matrine (Greenex Ultra® SL, $5 \mathrm{~g} / \mathrm{l}^{-1}$, Montana S. A., Perú). Las concentraciones usadas para spinetoram fueron de: $0,125 \mathrm{ml}^{\prime} 500 \mathrm{ml}^{-1}\left(0,075 \mathrm{l} / \mathrm{ha}^{-1}\right)$ y 0,375 
$\mathrm{ml} / 500 \mathrm{ml}^{-1}\left(0,3 \mathrm{l} / \mathrm{ha}^{-1}\right)$, y las concentraciones para matrine fueron de: $0,5 \mathrm{ml}^{-} 500 \mathrm{ml}^{-1}\left(200 \mathrm{ml} / 200 \mathrm{l}^{-1}\right) \mathrm{y}$ $1,5 \mathrm{ml}^{1} 500 \mathrm{ml}^{-1}\left(600 \mathrm{ml}^{-} 200 \mathrm{l}^{-1}\right)$ en base a los productos comerciales de uso agrícola.

\section{Ensayos de inmersión}

Los huevos y pupas de 24 h y 48 h de edad, respectivamente, se sumergieron durante tres segundos en soluciones insecticidas de ambos productos y dosis (Liu y Chen, 2000). Luego fueron colocados sobre papel toalla por 30 min para permitir el secado. Seguidamente, se transfirieron individualmente al interior de envases plásticos (12 ml de capacidad) y se mantuvieron bajo condiciones de oscuridad y ventilación. Se emplearon 10 huevos/pupas por cada tratamiento y con cuatro repeticiones incluyendo el control. Las lecturas se realizaron hasta el $80 \%$ de eclosión de huevos (120 h) y $80 \%$ de emergencia de adultos (> 120 h) en el control (lannacone et al., 2017).

\section{Ensayos de contacto residual}

Se realizaron aplicaciones de $0,5 \mathrm{ml}$ de la solución insecticida, con la ayuda de una jeringa desechable de $1 \mathrm{ml}$ de capacidad, en el interior de los envases plásticos y se esparció con un hisopo estéril por todas las paredes internas. Luego, se procedió a secar a temperatura ambiente durante 2 h. Seguidamente, se colocaron individualmente en cada envase 10 larvas del primer, segundo y tercer estadio y 10 adultos de $C$. externa y $C$. cincta, ambos estados de desarrollo con una edad entre 48-72 h, por cada tratamiento y con cuatro repeticiones incluyendo el control. Las lecturas de mortalidad fueron a las 24, 48 y $72 \mathrm{~h}$, mientras que para los adultos fueron a las 24 y 48 h de exposición (Alegre, lannacone y Carhuapoma, 2017).

\section{Ensayos de ingestión}

Los ensayos de ingestión en larvas se realizaron suministrando huevos de S. cerealella, los cuales fueron previamente sumergidos en las soluciones insecticidas durante $10 \mathrm{~s}$. Luego, fueron colocados sobre papel toalla por 10 min para permitir su secado, y seguidamente fueron pegados $1,0 \mathrm{~g}$ de huevos con un gel adhesivo no tóxico a cartulinas de $10 \times 10 \mathrm{~mm}$, las cuales se colocaron individualmente junto con una larva en el interior de un envase plástico (lannacone y Lamas, 2002). Se emplearon 10 larvas del primer, segundo y tercer estadio de $C$. externa y $C$. cincta de 48-72 h de edad, por cada tratamiento y con cuatro repeticiones incluyendo el control. Las lecturas de mortalidad se registraron a las 24, 48 y 72 h de exposición.

Los ensayos de ingestión en adultos se desarrollaron suministrando las soluciones insecticidas en frascos bebederos (con un paño esponja en la abertura). Un bebedero (frasco de vidrio de $5 \mathrm{ml}$ de capacidad) y 10 adultos de $C$. externay $C$. cincta de 48-96 h de edad, sin distinción de sexo, se introdujeron en un recipiente plástico $(30 \times 18 \times 7 \mathrm{~cm})$. Cada uno de los recipientes conformó una repetición, siendo en total cuatro repeticiones por cada tratamiento, incluyendo el control, el cual consistió de agua embotellada pura $(\mathrm{pH}=7,7)$. Las lecturas de mortalidad se realizaron a las 24, 48 y 72 h de exposición (Huerta, Medina, Budia y Viñuela, 2004).

\section{Diseño experimental}

Se utilizaron la máxima y mínima concentración recomendada en campo de cada producto químico, siendo para spinetoram: 0,125 ml/500 $\mathrm{ml}^{-1}(0,075 \mathrm{l} /$ ha $^{-1}$, calculado en referencia a un cilindro y medio) y $0,375 \mathrm{ml} / 500 \mathrm{ml}^{-1}\left(0,3 \mathrm{l} / \mathrm{ha}^{-1}\right.$, calculado en referencia a dos cilindros); y para matrine: $0,5 \mathrm{ml}^{-500 \mathrm{ml}^{-1}}$ (200 ml/200 l-1) y $1,5 \mathrm{ml}^{-} 500 \mathrm{ml}^{-1}\left(600 \mathrm{ml}^{-} 200 \mathrm{l}^{-1}\right)$. Para cada uno de los ensayos planteados, se realizaron cuatro repeticiones por cada tratamiento incluyendo el control (agua embotellada), el cual no debió exceder el $10 \%$ de mortalidad (Alegre et al., 2017). Los ensayos por inmersión, contacto residual e ingestión se desarrollaron con 10 individuos por cada concentración. Se consideraron muertos, a excepción de huevos y pupas, aquellos individuos que no exhibieron movimiento alguno, bajo observación en un estereoscopio.

\section{Análisis estadístico}

Se empleó un diseño completamente al azar (DCA) con cinco tratamientos (control, spinetoram $0,375 \mathrm{ml}^{\prime} / 500 \mathrm{ml}^{-1}$ y spinetoram $0,125 \mathrm{ml}^{-} / 500 \mathrm{ml}^{-1}, \mathrm{y}$ matrine $1,5 \mathrm{ml}^{-} 500 \mathrm{mll}^{-1} \mathrm{y}$ matrine $0,5 \mathrm{ml} / 500 \mathrm{ml}^{-1}$ ) con cuatro repeticiones. La eficacia de los tratamientos (\% de efecto) y las repeticiones se evaluaron a través de un análisis de varianza (ANOVA) de dos vías con prueba complementaria de significancia de Tukey; sin embargo, el ANOVA (prueba de homogeneidad de varianzas mediante el estadístico de Levene) no resultó significativo, a pesar de la transformación de los datos según la expresión log $(x+1)$, por lo que se recurrió a utilizar la prueba no paramétrica de Kruskall-Wallis en todos los ensayos toxicológicos. La mortalidad de los individuos 
(\%) se corrigió teniendo en cuenta la mortalidad natural que ocurrió en el control usando la fórmula propuesta por Schneider-Orelli (Alegre et al., 2017). Se usó el paquete estadístico IBM SPSS (versión 23), para el cálculo de los estadísticos a un nivel de significancia de $p<0,05$.

Los productos fitosanitarios se clasificaron utilizando los valores resultantes de acuerdo con las cuatro categorías de toxicidad para ensayos de laboratorio: $1=$ inofensivo ( $<30 \%$ de mortalidad), $2=$ ligeramente nocivo (30-79 \% de mortalidad), $3=$ moderadamente nocivo $(80-99 \%$ de mortalidad), 4= nocivo (> $99 \%$ de mortalidad) (Sterk et al., 1999).

\section{RESULTADOS Y DISCUSIÓN}

\section{Ensayos toxicológicos de inmersión}

Los porcentajes de eclosión obtenidos en los ensayos de inmersión de huevos y pupas de $C$. externa ( $p=0,09$ y $p=1,00$, respectivamente) y C. $\operatorname{cincta}(p=0,08$ y $p=0,38$, respectivamente) fueron estadísticamente similares al control. Ambos productos fitosanitarios resultaron inofensivos en ambos estados de desarrollo (Tabla 1). Los resultados presentados en este estudio fueron similares a los observados por Visnupriya y Muthukrishnan (2016), quienes obtuvieron una alta eclosión de huevos (entre el 81,6 - 95,2 \%) en Chrysoperla zastrowi sillemi (Esben-Petersen) con diferentes dosis de spinetoram. Al emplear la máxima concentración recomendada en campo de spinosad, producto fitosanitario del mismo grupo que el spinetoram, se obtuvo $0 \%$ de mortalidad de huevos de Chrysoperla carnea (Stephens) a las 24 h de exposición (El-Zahi, 2012). Asimismo, en otro estudio se demostró la selectividad del spinosad en huevos y pupas de $C$. carnea en condiciones de laboratorio (Medina, Budia, del Estal, Adan, y Viñuela, 2003a). La baja toxicidad del spinetoram y del matrine puede atribuirse a las barreras de protección natural que presentan los huevos (corión a prueba de agua) y pupas (capullo de seda), los cuales dificultan la penetración y absorción de los productos fitosanitarios (Medina, Budia, Tirry, Smagghe y Viñuela, 2001).

\section{Ensayos toxicológicos de contacto residual}

La Tabla 2 muestra los resultados de los ensayos por contacto residual en larvas de C. externa. Se observaron diferencias estadísticamente significativas respecto al spinetoram, en sus dos dosis respecto al control a las 24, 48 y 72 h en larvas del primer estadio de $C$. externa ( $p=0,003, p=0,004$ y $p=0,003$, respectivamente). El primer estadio de $C$. externa obtuvo las mayores mortalidades registradas a las $72 \mathrm{~h}$ con $42,11 \%$ y 28,95 \% con la máxima y mínima concentración de spinetoram. El segundo estadio larval obtuvo valores similares al control $(p=0,077)$ a las $24 \mathrm{~h}$, mientras que a las 48 y $72 \mathrm{~h}$ se observaron diferencias estadísticamente significativas respecto al control ( $p=0,005$ y $p=0,001$, respectivamente) con la máxima concentración del producto, el cual produjo la mayor mortalidad registrada en este estadio con $30 \%$. El tercer estadio larval de C. externa obtuvo a las $24 \mathrm{~h}$ valores estadísticamente similares al control $(p=1,000)$, mientras que los resultados obtenidos a las 48 y 72 h fueron estadísticamente diferentes

Tabla 1. Eclosión (\%) de huevos y emergencia (\%) de adultos mediante ensayos por inmersión en Chrysoperla externa y Ceraeochrysa cincta

\begin{tabular}{|c|c|c|c|c|c|c|c|c|}
\hline & \multicolumn{4}{|c|}{ Chrysoperla externa } & \multicolumn{4}{|c|}{ Ceraeochrysa cincta } \\
\hline Control & $\begin{array}{c}\text { Eclosión } \\
(\%) \\
100 a \\
\end{array}$ & Tox & $\begin{array}{c}\text { Emergencia } \\
(\%) \\
100 a \\
\end{array}$ & Tox & $\begin{array}{c}\text { Eclosión } \\
(\%) \\
100 a \\
\end{array}$ & Tox & $\begin{array}{c}\text { Emergencia } \\
(\%) \\
100 a \\
\end{array}$ & Tox \\
\hline \multicolumn{9}{|c|}{$\begin{array}{l}\text { Spinetoram } \\
\left(\mathrm{ml} / 500 \mathrm{ml}^{-1}\right)\end{array}$} \\
\hline 0,375 & $87,50 a$ & 1 & $100,00 a$ & 1 & $92,31 a$ & 1 & $95,00 a$ & 1 \\
\hline 0,125 & $92,50 a$ & 1 & $100,00 a$ & 1 & $94,87 a$ & 1 & $97,50 a$ & 1 \\
\hline \multicolumn{9}{|c|}{$\begin{array}{l}\text { Matrine } \\
\left(\mathrm{ml} / 500 \mathrm{ml}^{-1}\right)\end{array}$} \\
\hline 1,5 & $85,00 a$ & 1 & $100,00 a$ & 1 & $89,74 a$ & 1 & $97,50 a$ & 1 \\
\hline 0,5 & $95,00 a$ & 1 & $100,00 a$ & 1 & $97,44 a$ & 1 & $100,00 a$ & 1 \\
\hline$p$ & 0,09 & & 1,00 & & 0,08 & & 0,38 & \\
\hline
\end{tabular}

Letras minúsculas iguales en una misma columna representan que no hay diferencia significativa entre los valores por la prueba de Kruskal-Wallis $(p<0,05)$. Tox= Toxicidad. Catalogación toxicológica para ensayos de laboratorio de Sterk et al. $(1999)(1=$ inofensivo, $2=$ ligeramente nocivo, 3 = moderadamente nocivo, $4=$ nocivo). 
Tabla 2. Mortalidad (\%) del primer, segundo y tercer estadio larval de Chrysoperla externa por contacto residual

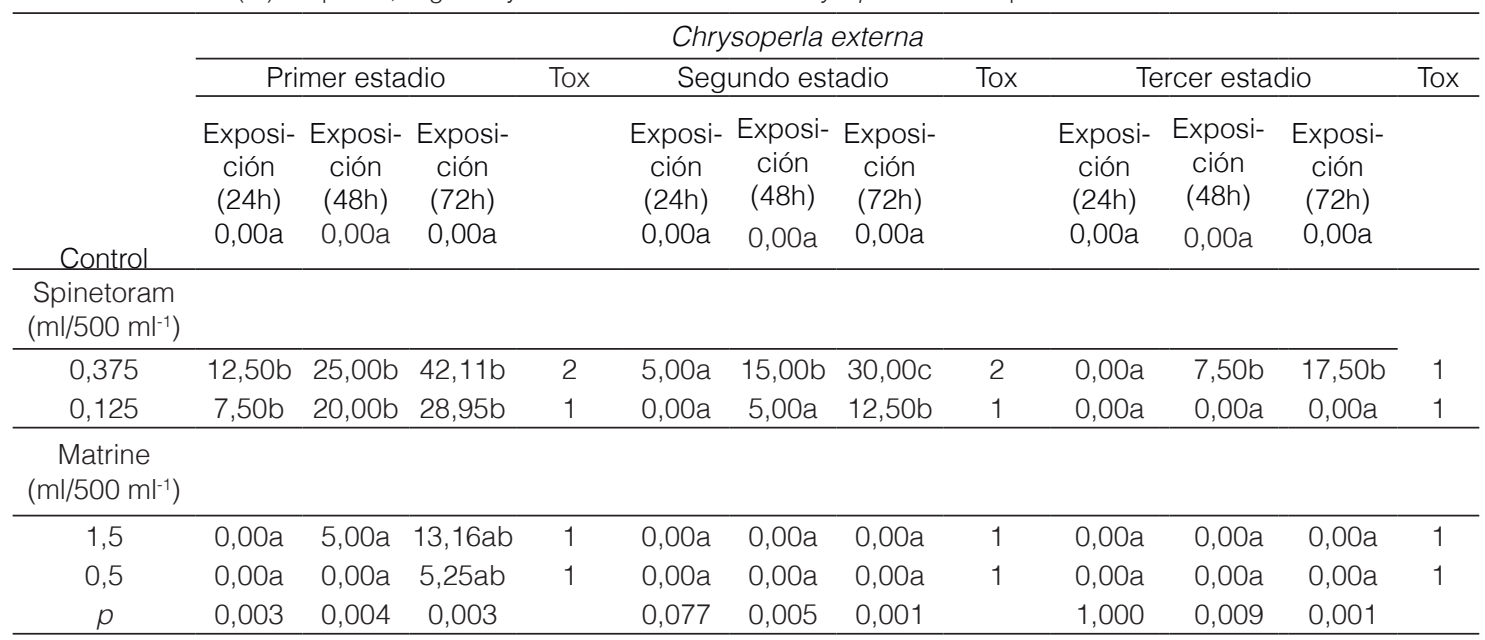

Letras minúsculas iguales en una misma columna representan que no hay diferencia significativa entre los valores por la prueba de Kruskal Wallis $(p<0,05)$. Tox= Toxicidad. Catalogación toxicológica para ensayos de laboratorio de Sterk et al. $(1999)(1=$ inofensivo, $2=$ ligeramente nocivo, $3=$ moderadamente nocivo, $4=$ nocivo)

al control ( $p=0,009$ y $p=0,001$, respectivamente) solo con la máxima concentración de spinetoram, el cual provocó la mayor mortalidad registrada en este estadio con $17,50 \%$. Los resultados obtenidos a las 24,48 y 72 h con la máxima y mínima concentración de matrine fueron similares al control en larvas del primer $(p=0,003 ; p=0,004$ y $p=0,003$, respectivamente), segundo ( $p=0,077$, $p=0,005$ y $p=0,001$, respectivamente) y tercer estadio ( $p=1,000 ; p=0,009$ y $p=0,001$, respectivamente). Según las categorías de toxicidad para ensayos de laboratorio, la máxima concentración de spinetoram resultó ligeramente nociva en larvas del primer y segundo estadio de $C$. externa a las $72 \mathrm{~h}$, mientras que en los demás casos ambos productos fitosanitarios fueron inofensivos a las 24, 48 y 72 h de exposición (Tabla 2).

La Tabla 3 muestra los resultados de los ensayos por contacto residual en larvas de C. cincta. No se observaron diferencias estadísticamente significativas respecto de los insecticidas evaluados, en sus dos dosis respecto al control $(p=0,388)$ a las $24 \mathrm{~h}$ en larvas del primer estadio de C. cincta. A las 48 y $72 \mathrm{~h}$ se observaron diferencias estadísticamente significativas respecto al control ( $p=0,048$ y $p=0,013$, respectivamente) con ambas concentraciones de los productos fitosanitarios, siendo la máxima concentración de spinetoram y matrine, los que ocasionaron las mayores mortalidades registradas en este estadio con 21,05 y $23,68 \%$, respectivamente a las $72 \mathrm{~h}$. Los valores obtenidos a las 24, 48 y $72 \mathrm{~h}$ en larvas del segundo ( $p=1,000 ; p=0,406$ y $p=0,406$, respectivamente) y tercer estadio $(p=1,000)$ fueron estadísticamente similares al control. Ambos productos fitosanitarios fueron inofensivos en todos los estadios larvales de C. cincta a las 24,48 y 72 h de exposición (Tabla 3).

Se ha demostrado la selectividad de las dosis recomendadas en campo de spinetoram al no presentar efectos nocivos en la población de enemigos naturales (Coccinella sp., Chrysopa sp. y Paederus sp.) en campos de cultivo de algodón (Elbarky, Hassan y El-Sayed, 2008). Por el contrario, en condiciones controladas, al evaluar la toxicidad con la máxima concentración de spinetoram $(131,1$ mg i.a/L) en larvas del segundo estadio de $C$. carnea y Chrysoperla johnsoni (Henry, Wells y Pupedis) por contacto directo, exposición residual e ingestión, ocasionó un porcentaje de mortalidad significativo solo en $C$. carnea con el $46,7 \pm 17,0$ a los 10 días después del tratamiento (Amarasekare y Shearer, 2013). Por el contrario, Visnupriya y Muthukrishnan (2016) obtuvieron mediante ensayos por contacto residual con spinetoram mortalidades que oscilaron entre 9,1-28,0 \% en larvas del segundo estadio de C. zastrowi sillemi (Esben-Petersen) a las $48 \mathrm{~h}$ de exposición. Los autores mencionados anteriormente indicaron la selectividad del producto en campo a diferencia de las condiciones controladas que presentaron niveles de toxicidad variable en crisópidos, resultado similar en este trabajo, lo que puede deberse a que el producto en condiciones ambientales puede ser degradado con mayor rapidez por agentes físicos o biológicos.

No existen muchos reportes científicos acerca de la toxicidad del producto fitosanitario matrine en crisópidos; sin embargo, Zhang, Dong y Du (2011) realizaron el único trabajo hasta la fecha empleando 
este producto en campo y concluyeron a lo largo de seis años de investigación mediante muestreos continuos que las densidades poblacionales de Chrysoperla sinica(Tjeder) fueron significativamente más altas en los campos de manejo orgánico de durazno tratados con matrine y piretrinas, en relación a los campos de manejo convencional de durazno.

La Tabla 4 presenta los resultados por contacto residual en adultos. Se observaron diferencias significativas respecto al control a las 24 y $48 \mathrm{~h}$ en adultos de $C$. externa $(p=0,005$ y $p=0,002$, respectivamente) y $C \cdot \operatorname{cincta}(p=0,007$ y $\mathrm{p}=0,002$, respectivamente) con ambas concentraciones de spinetoram, a excepción del valor obtenido con la mínima concentración de spinetoram en adultos de
$C$. cincta que fue estadísticamente similar al control $(p=0,007)$ a las $24 \mathrm{~h}$. Los resultados registrados con ambas concentraciones de matrine fueron estadísticamente similares al control a las 24 y 48 h en adultos de $C$. externa ( $p=0,005$ y $p=0,002$, respectivamente) y $C$. cincta ( $p=0,007$ y $p=0,002$, respectivamente). Según las categorías de toxicidad para ensayos de laboratorio, la máxima y mínima concentración de spinetoram fueron ligeramente nocivas para adultos de $C$. externa, mientras que para los adultos de $C$. cincta fue moderadamente nocivo y ligeramente nocivo, respectivamente. El matrine fue inofensivo en ambas especies a las 24 y $48 \mathrm{~h}$.

Roubos, Rodriguez-Saona, Holdcraft, Mason e Isaacs (2014), al evaluar el efecto residual de

Tabla 3. Mortalidad (\%) del primer, segundo y tercer estadio larval de Ceraeochrysa cincta por contacto residual

\begin{tabular}{|c|c|c|c|c|c|c|c|c|c|c|c|c|}
\hline \multirow{5}{*}{$\begin{array}{l}\text { Control } \\
\text { Spinetoram } \\
\text { (ml/500 ml- }\end{array}$} & \multicolumn{12}{|c|}{ Ceraeochrysa cincta } \\
\hline & \multicolumn{3}{|c|}{ Primer estadio } & \multirow[t]{3}{*}{ Tox } & \multicolumn{3}{|c|}{ Segundo estadio } & \multirow[t]{3}{*}{ Tox } & \multicolumn{3}{|c|}{ Tercer estadio } & \multirow[t]{3}{*}{ Tox } \\
\hline & $\begin{array}{c}\text { Exposi- } \\
\text { ción } \\
(24 h)\end{array}$ & $\begin{array}{c}\text { Exposi- } \\
\text { ción } \\
(48 h)\end{array}$ & $\begin{array}{l}\text { Exposi- } \\
\text { ción } \\
(72 h)\end{array}$ & & $\begin{array}{c}\text { Exposi- } \\
\text { ción } \\
\text { (24h) }\end{array}$ & $\begin{array}{l}\text { Exposi- } \\
\text { ción } \\
\text { (48h) }\end{array}$ & $\begin{array}{l}\text { Exposi- } \\
\text { ción } \\
(72 h)\end{array}$ & & $\begin{array}{c}\text { Exposi- } \\
\text { ción } \\
(24 h)\end{array}$ & $\begin{array}{l}\text { Exposi- } \\
\text { ción } \\
\text { (48h) }\end{array}$ & $\begin{array}{l}\text { Exposi- } \\
\text { ción } \\
\text { (72h) }\end{array}$ & \\
\hline & & 0,00a & 0,00a & & 0,00a & 0,00a & 0,00a & & 0,00a & & 0,00a & \\
\hline & & & & & & & & & & & & \\
\hline 0,375 & $5,00 a$ & $15,00 b$ & $21,05 b$ & 1 & $0,00 a$ & $0,00 a$ & $0,00 a$ & 1 & $0,00 a$ & $0,00 a$ & $0,00 a$ & 1 \\
\hline 0,125 & $2,50 a$ & $12,50 \mathrm{~b}$ & $15,79 b$ & 1 & $0,00 \mathrm{a}$ & $0,00 a$ & $0,00 a$ & 1 & $0,00 \mathrm{a}$ & $0,00 a$ & $0,00 a$ & 1 \\
\hline \multicolumn{13}{|c|}{$\begin{array}{l}\text { Matrine } \\
\left(\mathrm{ml} / 500 \mathrm{ml}^{-1}\right)\end{array}$} \\
\hline$\overline{1,5}$ & $7,50 a$ & $17,50 b$ & $23,68 b$ & 1 & $0,00 a$ & $2,50 a$ & $2,50 a$ & 1 & $0,00 a$ & $0,00 a$ & $0,00 a$ & 1 \\
\hline 0,5 & $0,00 a$ & $12,50 b$ & $18,42 b$ & 1 & $0,00 a$ & $0,00 a$ & $0,00 a$ & 1 & $0,00 \mathrm{a}$ & $0,00 \mathrm{a}$ & $0,00 a$ & 1 \\
\hline$p$ & 0,388 & 0,048 & 0,013 & & 1,000 & 0,406 & 0,406 & & 1,000 & 1,000 & 1,000 & \\
\hline
\end{tabular}

Letras minúsculas iguales en una misma columna representan que no hay diferencia significativa entre los valores por la prueba de Kruskal Wallis $(p<0,05)$. Tox= Toxicidad. Catalogación toxicológica para ensayos de laboratorio de Sterk et al. (1999) $(1=$ inofensivo $2=$ ligeramente nocivo, $3=$ moderadamente nocivo, $4=$ nocivo).

Tabla 4. Mortalidad (\%) de adultos de Chrysoperla externa y Ceraeochrysa cincta por contacto residual

\begin{tabular}{|c|c|c|c|c|c|c|}
\hline \multirow{3}{*}{ Control } & \multicolumn{2}{|c|}{$\begin{array}{c}\text { Chrysoperla externa } \\
\text { Exposición (h) }\end{array}$} & \multirow{3}{*}{ Tox } & \multicolumn{2}{|c|}{$\begin{array}{l}\text { Ceraeochrysa cincta } \\
\text { Exposición (h) }\end{array}$} & \multirow{3}{*}{ Tox } \\
\hline & 24 & 48 & & 24 & 48 & \\
\hline & $0,00 a$ & $0,00 a$ & & $0,00 \mathrm{a}$ & $0,00 a$ & \\
\hline \multicolumn{7}{|c|}{ Spinetoram $\left(\mathrm{ml} / 500 \mathrm{ml}^{-1}\right)$} \\
\hline 0,375 & $20,00 b$ & $72,50 b$ & 2 & $17,50 \mathrm{~b}$ & $80,00 b$ & 3 \\
\hline 0,125 & $12,50 b$ & $65,00 b$ & 2 & $7,50 a b$ & $60,50 b$ & 2 \\
\hline \multicolumn{7}{|c|}{ Matrine $\left(\mathrm{ml} / 500 \mathrm{ml}^{-1}\right)$} \\
\hline$\overline{1,5}$ & $0,00 a$ & $5,00 a$ & 1 & $0,00 a$ & $7,50 \mathrm{a}$ & 1 \\
\hline 0,5 & $0,00 a$ & 0,00a & 1 & $0,00 \mathrm{a}$ & $0,00 a$ & 1 \\
\hline$p$ & 0,005 & 0,002 & & 0,007 & 0,002 & \\
\hline
\end{tabular}

Letras minúsculas iguales en una misma columna representan que no hay diferencia significativa entre los valores por la prueba de Kruskal-Wallis $(p<0,05)$. Tox= Toxicidad. Catalogación toxicológica para ensayos de laboratorio de Sterk et al. (1999) $(1=$ inofensivo, $2=$ ligeramente nocivo, 3 = moderadamente nocivo, $4=$ nocivo). 
spinetoram, comprobaron a los 0 y 7 días un elevado porcentaje de mortalidad en adultos de Chrysoperla rufilabris (Burmeister), mientras que a los 14 días el producto químico presentó una menor toxicidad, lo cual pudo deberse a la degradación natural del producto. Por otro lado, se ha comprobado efectos agudos con la máxima concentración de spinetoram, siendo más perjudiciales para los adultos que para las larvas del segundo estadio de C. carnea (Amarasekare, Shearer y Mills, 2016). Ullah et al. (2017) demostraron que los adultos de C. carnea fueron más susceptibles al spinetoram en comparación al estadio larval por contacto residual, obteniendo un 93,33\% de mortalidad a las 48 h de exposición, resultados similares a este trabajo en condiciones de laboratorio que evidencian la alta susceptibilidad de este estado de desarrollo frente a este producto.

Es necesario evaluar estos resultados en condiciones de campo debido a que los niveles de los residuos de los productos fitosanitarios en las superficies vegetales pueden variar a diferentes condiciones ambientales, por la estructura de los cultivos, la calidad de la aplicación y el nivel de contacto o llegada del producto a las distintas partes de la planta.

\section{Ensayos toxicológicos de ingestión}

Las Tablas 5 y 6 presentan los resultados de los ensayos por ingestión en larvas de $C$. externa y $C$. cincta. No se observaron diferencias estadísticamente significativas respecto de los insecticidas evaluados, en sus dos dosis respecto al control a las 24,48 y 72 h en larvas del primer, segundo y tercer estadio de $C$. externa $(p=1,000, p=1,000, p=0,406 ; p=1,000 ; p=1,000$, respectivamente) y $C$. cincta $(p=0,406, p=0,116$, $p=0,116 ; p=1,000$ y $p=1,000$, respectivamente). Según la IOBC, la máxima y mínima concentración de spinetoram y matrine fueron inofensivos en ambos crisópidos. Sabry, Hassan y Rahman (2014) concluyeron que las larvas del segundo estadio de C. carnea, alimentadas con huevos tratados de $S$. cerealella con la máxima concentración de spinetoram (30 ppm), presentaron un porcentaje

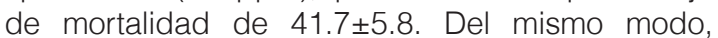
Visnupriya y Muthukrishnan (2016) al suministrar huevos tratados de Corcyra cephalonica Stainton (Lepidoptera: Pyralidae) con las dosis de 36, 45, 54 y $108 \mathrm{~g}$ i.a/ha de spinetoram en larvas del segundo estadio de $C$. zastrowisillemi, obtuvieron mortalidades que oscilaron entre 10,8 - 22,3 \% a las $48 \mathrm{~h}$. Los resultados obtenidos por los autores mencionados anteriormente no coinciden y no reflejan similitudes a este trabajo debido a los altos porcentajes de mortalidad registrados, pudiéndose inferir que la susceptibilidad de las especies en la familia Chrysopidae es variable.

La Tabla 7 presenta los resultados del ensayo toxicológico por ingestión en adultos. No se observaron diferencias significativas de los insecticidas respecto al control $(p=1,000)$ a las $24 \mathrm{~h}$ en adultos de $C$. externa y $C$. cincta con la máxima y mínima concentración de ambos productos fitosanitarios. A las $48 \mathrm{~h}$ se observaron diferencias significativas de las dosis de

Tabla 5. Mortalidad (\%) del primer, segundo y tercer estadio larval de Chrysoperla externa mediante ensayos de ingestión

\begin{tabular}{|c|c|c|c|c|c|c|c|c|c|c|c|c|}
\hline \multirow[b]{3}{*}{ Control } & \multicolumn{12}{|c|}{ Chrysoperla externa } \\
\hline & \multicolumn{3}{|c|}{ Primer estadio } & \multirow[t]{2}{*}{ Tox } & \multicolumn{3}{|c|}{ Segundo estadio } & \multirow[t]{2}{*}{ Tox } & \multicolumn{3}{|c|}{ Tercer estadio } & \multirow[t]{2}{*}{ Tox } \\
\hline & $\begin{array}{c}\text { Exposi- } \\
\text { ción } \\
(24 \mathrm{~h}) \\
0,00 \mathrm{a}\end{array}$ & $\begin{array}{c}\text { Exposi- } \\
\text { ción } \\
(48 \mathrm{~h}) \\
0,00 \mathrm{a}\end{array}$ & $\begin{array}{c}\text { Exposi- } \\
\text { ción } \\
\text { (72h) } \\
0,00 \mathrm{a}\end{array}$ & & $\begin{array}{c}\text { Exposi- } \\
\text { ción } \\
(24 \mathrm{~h}) \\
0,00 \mathrm{a}\end{array}$ & $\begin{array}{c}\text { Exposi- } \\
\text { ción } \\
(48 \mathrm{~h}) \\
0,00 \mathrm{a}\end{array}$ & $\begin{array}{c}\text { Exposi- } \\
\text { ción } \\
\text { (72h) } \\
0,00 \mathrm{a}\end{array}$ & & $\begin{array}{c}\text { Exposi- } \\
\text { ción } \\
(24 h) \\
0,00 a\end{array}$ & $\begin{array}{l}\text { Exposi-E } \\
\text { ción } \\
(48 \mathrm{~h}) \\
0,00 a\end{array}$ & $\begin{array}{l}\text { Exposi- } \\
\text { ción } \\
(72 \mathrm{~h}) \\
0,00 \mathrm{a}\end{array}$ & \\
\hline \multicolumn{13}{|c|}{$\begin{array}{l}\text { Spinetoram } \\
\left(\mathrm{ml}^{\prime} / 500 \mathrm{ml}^{-1}\right)\end{array}$} \\
\hline 0,375 & $0,00 a$ & $0,00 a$ & $2,50 a$ & 1 & $0,00 a$ & $0,00 a$ & $0,00 a$ & 1 & $0,00 a$ & $0,00 a$ & $0,00 a$ & 1 \\
\hline$\underline{0,125}$ & $0,00 a$ & $0,00 a$ & $0,00 a$ & 1 & $0,00 a$ & $0,00 a$ & $0,00 \mathrm{a}$ & 1 & $0,00 a$ & $0,00 a$ & $0,00 a$ & 1 \\
\hline \multicolumn{13}{|c|}{$\begin{array}{l}\text { Matrine } \\
\left(\mathrm{ml} / 500 \mathrm{ml}^{-1}\right)\end{array}$} \\
\hline 1,5 & $0,00 a$ & $0,00 a$ & $5,00 a$ & 1 & $0,00 a$ & $0,00 a$ & $0,00 a$ & 1 & $0,00 a$ & $0,00 a$ & $0,00 a$ & 1 \\
\hline 0,5 & $0,00 a$ & $0,00 a$ & $0,00 a$ & 1 & $0,00 a$ & $0,00 a$ & $0,00 a$ & 1 & $0,00 a$ & $0,00 \mathrm{a}$ & $0,00 \mathrm{a}$ & 1 \\
\hline$p$ & 1,000 & 1,000 & 0,406 & & 1,000 & 1,000 & 1,000 & & 1,000 & 1,000 & 1,000 & \\
\hline
\end{tabular}

Letras minúsculas iguales en una misma columna representan que no hay diferencia significativa entre los valores por la prueba de Kruskal-Wallis $(p<0,05)$. Tox = Toxicidad. Catalogación toxicológica para ensayos de laboratorio de Sterk et al. (1999) $(1=$ inofensivo, $2=$ ligeramente nocivo, $3=$ moderadamente nocivo, $4=$ nocivo). 
Tabla 6. Mortalidad (\%) del primer, segundo y tercer estadio larval de Ceraeochrysa cincta mediante ensayos de ingestión

\begin{tabular}{|c|c|c|c|c|c|c|c|c|c|c|c|c|}
\hline \multirow[b]{3}{*}{ Control } & \multicolumn{12}{|c|}{ Ceraeochrysa cincta } \\
\hline & \multicolumn{3}{|c|}{ Primer estadio } & \multirow[t]{2}{*}{ Tox } & \multicolumn{3}{|c|}{ Segundo estadio } & \multirow[t]{2}{*}{ Tox } & \multicolumn{3}{|c|}{ Tercer estadio } & \multirow[t]{2}{*}{ Tox } \\
\hline & $\begin{array}{c}\text { Exposi- } \\
\text { ción } \\
(24 h) \\
0,00 a\end{array}$ & $\begin{array}{c}\text { Exposi- } \\
\text { ción } \\
(48 \mathrm{~h}) \\
0,00 \mathrm{a}\end{array}$ & $\begin{array}{c}\text { Exposi- } \\
\text { ción } \\
(72 \mathrm{~h}) \\
0,00 \mathrm{a}\end{array}$ & & $\begin{array}{c}\text { Exposi- } \\
\text { ción } \\
(24 \mathrm{~h}) \\
0,00 \mathrm{a}\end{array}$ & $\begin{array}{c}\text { Exposi- } \\
\text { ción } \\
(48 \mathrm{~h}) \\
0,00 \mathrm{a}\end{array}$ & $\begin{array}{c}\text { Exposi- } \\
\text { ción } \\
(72 \mathrm{~h}) \\
0,00 \mathrm{a}\end{array}$ & & $\begin{array}{c}\text { Exposi- } \\
\text { ción } \\
(24 \mathrm{~h}) \\
0,00 \mathrm{a}\end{array}$ & $\begin{array}{c}\text { Exposi- } \\
\text { ción } \\
(48 \mathrm{~h}) \\
0,00 \mathrm{a}\end{array}$ & $\begin{array}{c}\text { Exposi- } \\
\text { ción } \\
(72 \mathrm{~h}) \\
0,00 \mathrm{a}\end{array}$ & \\
\hline \multicolumn{13}{|c|}{$\begin{array}{l}\text { Spinetoram } \\
\left(\mathrm{ml} / 500 \mathrm{ml}^{-1}\right)\end{array}$} \\
\hline 0,375 & $0,00 a$ & $7,50 a$ & $7,50 a$ & 1 & $0,00 \mathrm{a}$ & $0,00 a$ & $0,00 a$ & 1 & $0,00 a$ & $0,00 a$ & $0,00 a$ & 1 \\
\hline$\underline{0,125}$ & $0,00 \mathrm{a}$ & $2,50 \mathrm{a}$ & $2,50 \mathrm{a}$ & 1 & $0,00 \mathrm{a}$ & 0,00a & $0,00 \mathrm{a}$ & 1 & $0,00 \mathrm{a}$ & $0,00 \mathrm{a}$ & $0,00 \mathrm{a}$ & 1 \\
\hline \multicolumn{13}{|c|}{$\begin{array}{l}\text { Matrine } \\
\left(\mathrm{ml} / 500 \mathrm{ml}^{-1}\right)\end{array}$} \\
\hline 1,5 & $2,50 a$ & $7,50 a$ & $7,50 a$ & 1 & $0,00 a$ & $0,00 a$ & $0,00 \mathrm{a}$ & 1 & $0,00 a$ & $0,00 a$ & $0,00 a$ & 1 \\
\hline 0,5 & $0,00 \mathrm{a}$ & $0,00 a$ & $0,00 \mathrm{a}$ & 1 & $0,00 \mathrm{a}$ & $0,00 a$ & $0,00 \mathrm{a}$ & 1 & $0,00 \mathrm{a}$ & $0,00 a$ & $0,00 \mathrm{a}$ & 1 \\
\hline$p$ & 0,406 & 0,116 & 0,116 & & 1,000 & 1,000 & 1,000 & & 1,000 & 1,000 & 1,000 & \\
\hline
\end{tabular}

Letras minúsculas iguales en una misma columna representan que no hay diferencia significativa entre los valores por la prueba de Kruskal-Wallis $(p<0,05)$. Tox= Toxicidad. Catalogación toxicológica para ensayos de laboratorio de Sterk et al. (1999) $(1=$ inofensivo, $2=$ ligeramente nocivo, $3=$ moderadamente nocivo, $4=$ nocivo).

Tabla 7. Mortalidad (\%) de adultos de Chrysoperla externa y Ceraeochrysa cincta mediante ensayos de ingestión

\begin{tabular}{|c|c|c|c|c|c|c|c|c|}
\hline \multirow[b]{3}{*}{ Control } & \multicolumn{3}{|c|}{$\begin{array}{l}\text { Chrysoperla externa } \\
\text { Exposición (h) }\end{array}$} & \multirow{3}{*}{ Tox } & \multicolumn{3}{|c|}{$\begin{array}{l}\text { Ceraeochrysa cincta } \\
\text { Exposición (h) }\end{array}$} & \multirow{3}{*}{ Tox } \\
\hline & 24 & 48 & 72 & & 24 & 48 & 72 & \\
\hline & $0,00 a$ & $0,00 a$ & $0,00 a$ & & $0,00 a$ & $0,00 a$ & $0,00 a$ & \\
\hline \multicolumn{9}{|c|}{$\begin{array}{l}\text { Spinetoram } \\
\left(\mathrm{ml} / 500 \mathrm{ml}^{-1}\right)\end{array}$} \\
\hline 0,375 & $0,00 a$ & $22,50 b$ & $40,00 \mathrm{c}$ & 2 & $0,00 a$ & $27,50 c$ & $47,50 \mathrm{~b}$ & 2 \\
\hline 0,125 & $0,00 a$ & $12,50 b$ & $32,50 \mathrm{bc}$ & 2 & $0,00 a$ & $17,50 \mathrm{bc}$ & $40,00 b$ & 2 \\
\hline \multicolumn{9}{|c|}{$\begin{array}{l}\text { Matrine } \\
\left(\mathrm{ml} / 500 \mathrm{ml}^{-1}\right)\end{array}$} \\
\hline 1,5 & $0,00 a$ & $5,00 a b$ & $22,50 b$ & 1 & $0,00 a$ & $15,00 \mathrm{bc}$ & $30,00 b$ & 2 \\
\hline 0,5 & $0,00 a$ & 0,00a & $0,00 a$ & 1 & $0,00 a$ & $7,50 a b$ & $7,50 a$ & 1 \\
\hline$p$ & 1,000 & 0,008 & 0,002 & & 1,000 & 0,006 & 0,003 & \\
\hline
\end{tabular}

Letras minúsculas iguales en una misma columna representan que no hay diferencia significativa entre los valores por la prueba de Kruskal-Wallis $(p<0,05)$. Tox= Toxicidad. Catalogación toxicológica para ensayos de laboratorio de Sterk et al. (1999) $(1=$ inofensivo, $2=$ ligeramente nocivo, $3=$ moderadamente nocivo, $4=$ nocivo).

spinetoram en relación al control para $C$. externa $(p=0,008)$ y $C$. cincta $(p=0,006)$. Asimismo, el valor obtenido solo con la máxima concentración de matrine fue estadísticamente diferente al control $(p=0,006)$. A las $72 \mathrm{~h}$ se observaron diferencias significativas respecto al control con la máxima y mínima concentración de spinetoram y la máxima concentración de matrine para $C$. externa $(p=0,002)$ y $C$. $\operatorname{cincta}(p=0,003)$. Según la IOBC, el spinetoram fue ligeramente nocivo para $C$. externa y $C$. cincta, mientras que solo la máxima concentración de matrine fue ligeramente nociva para $C$. cincta a las $72 \mathrm{~h}$, siendo en los demás casos inofensivo.

Los adultos de $C$. carnea y $C$. johnsoni fueron muy susceptibles al spinetoram al evaluar la toxicidad del producto mediante ingestión, contacto directo y exposición residual, obteniendo mortalidades superiores al $90 \%$ en ambas especies a los 10 días después del tratamiento (Amarasekare y Shearer, 2013). Visnupriya y Muthukrishnan (2016) observaron que la longevidad de adultos de $C$. zastrowi sillemi fue entre 7,7 - 11,4 días mediante ensayos de ingestión de una solución de sacarosa al $10 \%$ tratada con spinetoram. Medina et al. (2003a) obtuvieron con la máxima concentración de spinosad el $87,5 \%$ de mortalidad de adultos de C. carnea. Los ensayos de ingestión con 800 y $400 \mathrm{mg} / \mathrm{i} . \mathrm{a} / \mathrm{L}^{-1}$ de spinosad alcanzaron una mortalidad del $100 \%$ de adultos de C. carnea a las 96 h de exposición (Medina, Budia, del Estal y 
Viñuela, 2003b). Por el contrario, al suministrar en agua potable el spinosad en adultos de $C$. externa, obtuvieron una mayor toxicidad de este producto (Rimoldi, Schneider y Ronco, 2012). La mayoría de los resultados mencionados anteriormente por los autores son coincidentes con los presentados en este estudio, evidenciando nuevamente la susceptibilidad del estado adulto de C. externa y C. cincta.

Si bien no existen muchos registros de autores que aborden la toxicidad o selectividad de estos productos fitosanitarios en crisópidos, los resultados presentados en este trabajo proporcionan una importante base científica para el uso adecuado del spinoteram y del matrine, y la protección y la utilización de los predadores $C$. externa y $C$. cincta en futuros ensayos en campo en el marco de un MIP. Es necesario continuar evaluando estos dos productos fitosanitarios mediante pruebas en campos agrícolas, donde las condiciones ambientales no son controlados y en la que la heterogeneidad del ambiente puede influir variando la supervivencia de los crisópidos y la "selectividad" ambiental del spinoteram y del matrine.

\section{CONCLUSIONES}

Los productos fitosanitarios usados en el presente trabajo fueron selectivos (inofensivos) en huevos y pupas de $C$. externa y $C$. cincta. Los primeros estadios larvales de $C$. externa y C. cincta fueron más susceptibles en relación al segundo y tercer estadio larval en los ensayos por contacto residual; sin embargo, fueron los adultos de ambos crisópidos los que registraron los más altos porcentajes de mortalidad con la máxima concentración de spinetoram. Mediante ensayos de ingestión, los adultos de $C$. externa y $C$. cincta fueron más susceptibles en relación al estado larval con ambos insecticidas. Se sugiere realizar liberaciones en campo de huevos y larvas de $C$. externa y $C$. cincta en cultivos donde se apliquen estos productos fitosanitarios, debido a que estos estados de desarrollo fueron los más tolerantes frente a las dosis recomendadas de spinetoram y matrine. Finalmente, en cuanto a la preservación de adultos de $C$. externa y $C$. cincta en campo: a pesar de la alta susceptibilidad de este estado de desarrollo frente al spinetoram observada en laboratorio, gracias a la resistencia de estas especies frente a diversos insecticidas y la casi nula probabilidad de alimentación de néctares contaminados con estos productos, estas especies pueden prosperar en un MIP aplicado en determinados agroecosistemas del país.

\section{BIBLIOGRAFÍA}

Alegre, A., lannacone, J. y Carhuapoma, M. (2017). Toxicidad del extracto acuoso, etanólico y hexánico de Annona muricata, Minthostachys mollis, Lupinus mutabilis, y Chenopodium quinoa sobre Tetranychus urticae y Chrysoperla externa. Chilean Journal of Agricultural \& Animal Sciences, 33(3), 273-284. DOI: https://dx.doi.org/10.4067/S0719-38902017005000705

Amarasekare, K. G. y Shearer, P. W. (2013). Comparing effects of insecticides on two green lacewings species, Chrysoperla johnsoni and Chrysoperla carnea (Neuroptera: Chrysopidae). Journal of Economic Entomology, 106(3), 1126-1133. DOI: https://doi. org/10.1603/EC12483

Amarasekare, K. G., Shearer, P. W. y Mills, N. J. (2016). Testing the selectivity of pesticide effects on natural enemies in laboratory bioassays. Biological Control, 102, 7-16. DOI: https://doi.org/10.1016/j. biocontrol.2015.10.015

Baker, B. P., Green, T. A. y Loker, A. J. (2020). Biological control and integrated pest management in organic and conventional systems. Biological Control, 140, 104095. DOI: https://doi.org/10.1016/j.biocontrol.2019.104095

Bueno, A. F., Carvalho, G. A., Santos, A. C., Sosa-Gómez, D. R. y Silva, D. M. (2017). Pesticide selectivity to natural enemies: challenges and constraints for research and field recommendation. Ciência Rural, 47(6), e20160829. DOI: https://doi.org/10.1590/0103$8478 \mathrm{cr} 20160829$

Depalo, L., Lanzoni, A., Masetti, A., Pasqualini, E. y Burgio, G. (2017). Lethal and sub-lethal effects of four insecticides on the aphidophagous coccinellid Adalia bipunctata (Coleoptera: Coccinellidae). Journal of Economic Entomology, 110(6), 2662-2671. DOI: https:// doi.org/10.1093/jee/tox243

Dripps, J. E., Boucher, R. E., Chloridis, A., Cleveland, C. B., DeAmicis, C. V., Gomez, L. E., ... Watson, G. B. (2011). The Spinosyn Insecticides. En O. Lopez y J. G. Fernandez-Bolanos (Eds.), Green Trends in Insect Control (163-212). Cambridge, Reino Unido: Royal Society of Chemistry. DOI: https://doi. org/10.1039/9781849732901-00163

Du, Y., Li, J., He, B. y Liu, A. (2004). Effects of matrine on the sodium channel in central neurons of the cotton bollworm, Helicoverpa armigera. Acta Entomologica Sinica, 47(2), 189-192. Recuperado de: https:// europepmc.org/article/cba/399141\#impact

Elbarky, N. M., Hassan, F. D. y El-Sayed, Y. A. (2008). Toxicological evaluation and biochemical impacts for radiant as a new generation of spinosyn on Spodoptera littoralis (Boisd.) larvae. Egyptian Academic Journal of Biological Sciences, 1(2), 85-97. DOI: https://doi. org/10.21608/eajbsa.2008.15738 
El-Zahi, E. (2012). Selectivity of some pesticides for various stages of Chrysoperla carnea (Steph.) using different methods of exposure. Egyptian Journal of Biological Pest Control, 22(2), 211-216. Recuperado de: https:// www.semanticscholar.org/paper/Selectivity-of-somepesticides-for-various-stages-El-Zahi/ef7347e8f03a97c 3fb5d07d6555c4a1c2b2dbab5

Fu, B., Li, Q., Qiu, H., Tang, L., Zeng, D., Liu, K. y Gao, Y. (2018). Resistance development, stability, cross-resistance potential, biological fitness and biochemical mechanisms of spinetoram resistance in the Thrips hawaiiensis (Thysanoptera: Thripidae). Pest Management Science, 74(7), 1564-1574. DOI: https:// doi.org/10.1002/ps.4887

Huang, J. y Xu, H. (2016). Matrine: Bioactivities and structural modifications. Current Topics in Medicinal Chemistry, 16(28), 3365-3378. DOI: https://doi.org/10. 2174/1568026616666160506131012

Huerta, A., Medina, P., Budia, F. y Viñuela, E. (2004). Evaluación de la toxicidad por ingestión de cuatro insecticidas y el colorante Floxín-B en larvas y adultos de Chrysoperla carnea (Stephens) (Neuroptera: Chrysopidae). Boletín de Sanidad Vegetal - Plagas, 30, 721-732. Recuperado de: https://www.mapa. gob.es/ministerio/pags/Biblioteca/Revistas/pdf_ plagas\%2FBSVP-30-04-721-732.pdf

lannacone, J. y Lamas, G. (2002). Efecto de dos extractos botánicos y un insecticida convencional sobre el depredador Chrysoperla externa. Manejo Integrado de Plagas y Agroecología, 65, 92-101. Recuperado de: http://repositorio.bibliotecaorton.catie.ac.cr/handle/ $11554 / 6100$

lannacone, J., Alvariño, L., Guabloche, A., Ventura, K., La Torre, M. I., Carhuapoma, M. y Castañeda, L. (2017). Efecto tóxico agudo y crónico de Tagetes minuta "huacatay" (asteraceae) y carbaril sobre seis entomófagos de importancia en control biológico. The Biologist (Lima), 15, 85-97. DOI: https://doi. org/10.24039/rtb2017151145

IBM SPSS Statistics for Windows (Version 23) [Software]. Armonk, Nueva York: IBM Corp.

Jindal, V., Dhaliwal, G. S. y Koul, O. (2013). Pest Management in $21^{\text {st }}$ Century: Roadmap for Future. Biopesticides International, 9, 1-22. Recuperado de: https://www. researchgate.net/publication/270276481_Pest_ Management_in_21st_Century_Roadmap_for_Future

Liu, T. X. y Chen, T. Y. (2000). Effects of the chitin synthesis inhibitor buprofezin on survival and development of immatures of Chrysoperla rufilabris (Neuroptera: Chrysopidae). Journal of Economic Entomology, 93(2), 234-239. DOI: https://doi.org/10.1603/0022-049393.2.234

Medina, P., Budia, F., Tirry, L., Smagghe, G. y Viñuela, E. (2001). Compatibility of spinosad, tebufenozide and azadirachtin with eggs and pupae of the predator Chrysoperla carnea (Stephens) under laboratory conditions. Biocontrol Science and Technology, 11 (5), 597-610. DOI: https://doi. org/10.1080/09583150120076157

Medina, P., Budia, F., del Estal, P., Adan, A. y Viñuela, E. (2003a). Side-effects of six insecticides on different developmental stages of Chrysoperla carnea (Neuroptera: Chrysopidae). OILB/wprs Bulletin, 26(5), 33-40. Recuperado de: https://www. researchgate.net/publication/230701720_OlLBwprs_ Bulletin_26_5_33-40_2003_Side-effects_of_six_ insecticides_on_different_developmental_stages_of_ Chrysoperla_carnea_Neuroptera_Chrysopidae

Medina, P., Budia, F., del Estal, P. y Viñuela, E. (2003b). Effects of three modern insecticides, pyriproxyfen, spinosad and tebufenozide, on survival and reproduction of Chrysoperla carnea adults. Annals of Applied Biology, 142, 55-61. DOI: https://doi. org/10.1111/j.1744-7348.2003.tb00229.x

Núñez, E. (1988a). Ciclo biológico y crianza de Chrysoperla externa y Ceraeochrysa cincta (Neuroptera, Chrysopidae). Revista Peruana de Entomología, 31, 76-82. Recuperado de: https://sisbib.unmsm.edu.pe/ BVRevistas/entomologia/v31/pdf/a16v31.pdf

Núñez, E. (1988b). Chrysopidae (Neuroptera) del Perú y sus especies más comunes. Revista Peruana de Entomología, 31, 69-75. Recuperado de: https://sisbib. unmsm.edu.pe/BVRevistas/entomologia/v31/pdf/ a15v31.pdf

Rimoldi, F., Schneider, M. I. y Ronco, A. E. (2012). Short and long-term effects of endosulfan, cypermethrin, spinosad, and methoxyfenozide on adults of Chrysoperla externa (Neuroptera: Chrysopidae). Journal of Economic Entomology, 105(6), 1982-1987. DOI: https://doi.org/doi:10.1603/ec12189

Roubos, C. R., Rodriguez-Saona, C., Holdcraft, R., Mason, K. S. e Isaacs, R. (2014). Relative toxicity and residual activity of insecticides used in blueberry pest management: mortality of natural enemies. Journal of Economic Entomology, 107, 277-285. DOI: https://doi. org/10.1603/ec13191

Sabry, A. H., Hassan, K. A. y Rahman, A. A. (2014). Relative toxicity of some modern insecticides against the pink bollworm, Pectinophora gossypiella (Saunders) and their residues effects on some natural enemies. International Journal of Science, Environment and Technology, 3 (2), 481-491. Recuperado de: http:// www.ijset.net/journal/274.pdf

Servicio Nacional de Sanidad Agraria (SENASA) (2015). Manual de crianza de insectos benéficos del 2015. Lima, Perú: Servicio Nacional de Sanidad Agraria, Subdirección del Control Biológico. Recuperado de: https://www.senasa.gob.pe/ 
senasa/descargasarchivos/2017/09/Gu\%C3\%ADaPr\%C3\%A1ctica-producci\%C3\%B3n-InsectosBen\%C3\%A9ficos.pdf

Sistema Integrado de Gestión de Insumos Agropecuarios (SIGIA) (2009). Consultas del registro de plaguicidas. Recuperado de: https://servicios.senasa.gob.pe/ SIGIAWeb/sigia_consulta_cultivo.html

Sterk, G., Hassan, S. A., Baillod, M., Bakker, F., Bigler, F., Blümel, S., ... Vogt, H. (1999). Results of the seventh joint pesticide testing programme carried out by the IOBCMPRS - Working Group "Pesticides and Beneficial Organisms". BioControl, 44, 99-117. DOI: https://doi.org/10.1023/a:1009959009802

Topping, C. J., Aldrich, A. y Berny, P. (2020). Overhaul environmental risk assessment for pesticides. Science, 367(6476), 360-363. DOI: https://doi.org/10.1126/ science.aay 1144

Ullah, Z., Sabri, M. A., Ahmad, S., Bilal, H., Hussain,
D., Mughal, T. K., ... Ahmad, M. I. (2017). In vitro study of comparative toxicity of different insecticides against Chrysoperla carnea (Stephens) (Neuroptera: Chrysopidae). Journal of Entomology and Zoology Studies, 5(3), 697-702. Recuperado de: http://www. entomoljournal.com/archives/?year $=2017 \& \mathrm{vol}=5 \&$ issu $\mathrm{e}=3 \&$ Articleld $=1875$

Visnupriya, M. y Muthukrishnan, N. (2016). Impact of natural toxin spinetoram 12 SC w/v (11.7 w/w) against Trichogramma chilonis Ishii and Chrysoperla zastrowi sillemi (Esben-Petersen) under laboratory conditions. African Journal of Agricultural Research, 11 (25), 22242230. DOI: https://doi.org/10.5897/AJAR2014.9085

Zhang, D. W., Dong, M. y Du, X. (2011). Effects of organic management on predator densities of Myzus persicae (Sulzer) during transition to organic agriculture in peach fields in China. Journal of Sustainable Agriculture, 35(8), 826-839. DOl:https://doi.org/10.1080/10440046. 2011.588997 